\title{
On Pseudo Cyclic Ricci Symmetric Manifolds
}

\author{
Shyamal Kumar Hui \\ Nikhil Banga Sikshan Mahavidyalaya Bishnupur, Bankura, 722122, West Bengal, India \\ e-mail: shyamal_hui@yahoo.co.in
}

Received:28 September 2010, Accepted: 28 April 2011

\begin{abstract}
The object of the present paper is to study concircularly symmetric $(P C R S)_{n}$, concircularly recurrent $(P C R S)_{n}$, decomposable $(P C R S)_{n}$. Among others it is shown that in a decomposable $(P C R S)_{n}$ one of the decompositions is Ricci flat and the other decomposition is cyclic parallel. The totally umbilical hypersurfaces of $(P C R S)_{n}$ are also studied.
\end{abstract}

Key words: Concircularly symmetric manifold, Concircularly recurrent manifold, Decomposable manifold, Pseudo cyclic Ricci symmetric manifold, Totally umbilical hypersurfaces.

Mathematics Subject Classification 2000: 53B30, 53B50, 53C15, 53C25.

\section{Yarı Devirli Ricci Simetrik Manifoldlar Üzerine}

Abstract: $\mathrm{Bu}$ makalenin amacı, konsirkular simetrik $(P C R S)_{n}$, konsirkular tekrarlı $(P C R S)_{n}$, ayrışabilir $(P C R S)_{n}$ manifoldları incelemektir. $(P C R S)_{n}$ ayrışabilir manifoldunda ayrışımlardan birisinin Ricci düzlemsellik (flat), diğerinin de devirli paralellik olduğu gösterilmiştir. Aynı zamanda $(P C R S)_{n}$ nin tümüyle umbilik hiperyüzeyleri çalışılmıştır.

Anahtar kelimeler: Konsirkular simetrik manifold, Konsirkular tekrarlı manifold, Ayrışabilir manifold, Yarı devirli Ricci simetrik manifold, Tümüyle umbilik hiperyüzeyler.

\section{Introduction}

A Riemannian manifold is Ricci symmetric if its Ricci tensor $S$ of type $(0,2)$ satisfies $\nabla S=0$, where $\nabla$ denotes the Riemannian connection. During the last five decades, the notion of Ricci symmetry has been weakened by many authors in several ways such as Ricci-recurrent manifolds [1], Ricci semi-symmetric manifolds [2], pseudo Ricci symmetric manifolds by M. C. Chaki [3]. A non-flat Riemannian manifold $\left(M^{n}, g\right)$ is said to be pseudo Ricci symmetric [3] if its Ricci tensor $S$ of type $(0,2)$ is not identically zero and satisfies the condition

$$
\left(\nabla_{X} S\right)(Y, Z)=2 A(X) S(Y, Z)+A(Y) S(Z, X)+A(Z) S(X, Y),
$$

where $A$ is a nowhere vanishing 1 -form. Such an $n$-dimensional manifold is denoted by $(P R S)_{n}$.

Extending the notion of pseudo Ricci symmetric manifold, recently A. A. Shaikh and the present author [4] introduced the notion of pseudo cyclic Ricci symmetric manifolds. A Riemannian manifold $\left(M^{n}, g\right)(n>2)$ is said to be pseudo cyclic Ricci symmetric 
manifold if its Ricci tensor $S$ of type $(0,2)$ is not identically zero and satisfies the following:

$$
\begin{aligned}
& \left(\nabla_{X} S\right)(Y, Z)+\left(\nabla_{Y} S\right)(Z, X)+\left(\nabla_{Z} S\right)(X, Y) \\
& \quad=2 A(X) S(Y, Z)+A(Y) S(X, Z)+A(Z) S(Y, X) \\
& \text { or } \\
& \left(\nabla_{X} S\right)(Y, Z)+\left(\nabla_{Y} S\right)(Z, X)+\left(\nabla_{Z} S\right)(X, Y) \\
& \quad=2 A(Y) S(Z, X)+A(Z) S(X, Y)+A(X) S(Y, Z) \\
& \text { or } \\
& \left(\nabla_{X} S\right)(Y, Z)+\left(\nabla_{Y} S\right)(Z, X)+\left(\nabla_{Z} S\right)(X, Y) \\
& \quad=2 A(Z) S(X, Y)+A(X) S(Y, Z)+A(Y) S(Z, X),
\end{aligned}
$$

where $A$ is a nowhere vanishing 1 -form associated to the vector field $\rho$ such that $A(X)=g(X, \rho)$ for all $X$. Such an $n$-dimensional manifold is denoted by $(P C R S)_{n}$. The $(P C R S)_{n}$ admitting semi-symmetric metric connection is also studied in [5]. The pseudo cyclic Ricci symmetric manifolds are also studied in $[6,7]$.

The object of the present paper is to study $(P C R S)_{n}$. The paper is organized as follows. Section 2 is devoted to the study of concircularly symmetric $(P C R S)_{n}$. It is shown that in a concircularly symmetric $(P C R S)_{n}$ with constant scalar curvature, $-r$ is an eigenvalue of the Ricci tensor $S$ corresponding to the eigenvector $\rho$. Section 3 deals with a study of concircularly recurrent $(P C R S)_{n}$. It is proved that in a concircularly recurrent $(P C R S)_{n}$ with constant scalar curvature, $-n$ is an eigenvalue of the Ricci tensor $S$ corresponding to the eigenvector $\rho$. In section 4 , we study decomposable $(P C R S)_{n}$ and it is shown that in a decomposable $(P C R S)_{n}$, one of the decompositions is Ricci flat and the Ricci tensor of the other decomposition is cyclic parallel.

Recently Özen and Altay [8] studied the totally umbilical hypersurfaces of weakly and pseudosymmetric spaces. Again Özen and Altay [9] also studied the totally umbilical hypersurfaces of weakly concircular and pseudo concircular symmetric spaces. In this connection it may be mentioned that Shaikh, Roy and Hui [10] studied the totally umbilical hypersurfaces of weakly conharmonically symmetric spaces. Section 5 deals with the study of totally umbilical hypersurfaces of $(P C R S)_{n}$. It is proved that the totally geodesic hypersurface of a $(P C R S)_{n}$ is also a $(P C R S)_{n}$.

\section{Concircularly Symmetric (PCRS)n}

A $(P C R S)_{n}$ is said to be concircularly symmetric if its concircular curvature tensor $\tilde{C}$, given by,

$$
\tilde{C}(Y, Z, U, V)=R(Y, Z, U, V)-\frac{r}{n(n-1)} G(Y, Z, U, V)
$$

where $r$ is the scalar curvature of the manifold and the tensor $G$ is defined by 
satisfies the relation

$$
G(Y, Z, U, V)=g(Z, U) g(Y, V)-g(Y, U) g(Z, V),
$$

$$
\left(\nabla_{X} \tilde{C}\right)(Y, Z, U, V)=0 .
$$

Let us consider a concircularly symmetric $(P C R S)_{n}$. Then by virtue of (3), it follows from (5) that

$$
\left(\nabla_{X} R\right)(Y, Z, U, V)-\frac{d r(X)}{n(n-1)} G(Y, Z, U, V)=0 .
$$

Let $\left\{e_{i}: i=1,2, \ldots, n\right\}$ be an orthonormal basis of the tangent space at any point of the manifold. Then putting $Y=V=e_{i}$ in (6) and taking summation over $i, 1 \leq i \leq n$, we get

$$
\left(\nabla_{X} S\right)(Z, U)=\frac{d r(X)}{n} g(Z, U) .
$$

Using (7) in (2), we obtain

$$
\begin{aligned}
& 2 A(X) S(Y, Z)+A(Y) S(X, Z)+A(Z) S(Y, X) \\
& \quad=\frac{1}{\mathrm{n}}[\operatorname{dr}(X) g(Y, Z)+\operatorname{dr}(Y) g(Z, X)+d r(Z) g(X, Y)] .
\end{aligned}
$$

Taking contraction of (8) over $Y$ and $Z$, we get

$$
A(Q X)+r A(X)=\frac{n+2}{2 n} d r(X),
$$

where $Q$ is the Ricci-operator i.e., $g(Q X, Y)=S(X, Y)$ for all $X, Y$.

We now suppose that the scalar curvature $r$ is constant, then

In view of (10), (9) yields

$$
d r(X)=0 \text { for all } X .
$$

i.e.,

$$
A(Q X)=-r A(X),
$$

$$
S(X, \rho)=-r g(X, \rho) .
$$

This leads to the following:

Theorem 2.1. In a concircularly symmetric $(P C R S)_{n}$ with constant scalar curvature, $-r$ is an eigenvalue of the Ricci tensor $S$ corresponding to the eigenvector $\rho$.

Since every concircularly flat manifold is concircularly symmetric. So by virtue of Theorem 2.1, we can state the following:

Corollary 2.1. In a concircularly flat $(P C R S)_{n}$ with constant scalar curvature, $-r$ is an eigenvalue of the Ricci tensor $S$ corresponding to the eigenvector $\rho$. 


\section{S.K. Hui}

\section{Concircularly Recurrent (PCRS)}

Definition 3.1. A $(P C R S)_{n}$ is said to be concircularly recurrent $([11,12])$ if its concircular curvature tensor $\tilde{C}$ satisfies the relation

$$
\left(\nabla_{X} \tilde{C}\right)(Y, Z, U, V)=A(X) \tilde{C}(Y, Z, U, V)
$$

where $A$ is a non-vanishing 1 -form.

We now consider a concircularly recurrent $(P C R S)_{n}$. Then by virtue of (3), it follows from (13) that

$$
\begin{aligned}
\left(\nabla_{X} R\right)(Y, Z, U, V)-\frac{d r(X)}{n(n-1)} G(Y, Z, U, V) \\
\quad=A(X)\left[R(Y, Z, U, V)-\frac{r}{n(n-1)} G(Y, Z, U, V)\right] .
\end{aligned}
$$

Contracting (14) over $Y$ and $V$, we get

$$
\left(\nabla_{X} S\right)(Z, U)-\frac{d r(X)}{n} g(Z, U)=A(X)\left[S(Z, U)-\frac{r}{n} g(Z, U)\right] .
$$

By virtue of (10), (15) yields

$$
\left(\nabla_{X} S\right)(Z, U)=A(X)\left[S(Z, U)-\frac{r}{n} g(Z, U)\right] .
$$

Using (16) in (2), we obtain

$$
A(X) S(Y, Z)=-\frac{r}{n}[A(X) g(Y, Z)+A(Y) g(Z, X)+A(Z) g(X, Y)] .
$$

Again taking contraction of (17) over $Y$ and $Z$, we get

$$
r[A(Q X)+n A(X)]=0 \quad \text { for all } X \text {. }
$$

Since the scalar curvature $r$ of $(P C R S)_{n}$ is always non-zero [4]. Therefore (3.6) yields

i.e.,

$$
A(Q X)=-n A(X)
$$

$$
S(X, \rho)=-n g(X, \rho) .
$$

Thus we can state the following:

Theorem 3.1. In a concircularly recurrent $(P C R S)_{n}$ with constant scalar curvature, $-n$ is an eigenvalue of the Ricci tensor $S$ corresponding to the eigenvector $\rho$.

\section{Decomposable (PCRS)}

A Riemannian manifold $\left(M^{n}, g\right)$ is said to be decomposable manifold [13] if it can be expressed as $M_{1}^{p} \times M_{2}^{n-p}$ for $2 \leq p \leq n$-2, that is, in some coordinate neighbourhood of the Riemannian manifold $\left(M^{n}, g\right)$, the metric can be expressed as 


$$
d s^{2}=g_{i j} d x^{i} d x^{j}=\bar{g}_{a b} d x^{a} d x^{b}+\stackrel{*}{*}_{\alpha \beta} d x^{\alpha} d x^{\beta},
$$

where $\bar{g}_{a b}$ are functions of $x^{1}, x^{2}, \ldots, x^{p}(p<n)$ denoted by $\bar{x}$ and $\stackrel{*}{g}_{\alpha \beta}$ are functions of $x^{p+1}, x^{p+2}, \ldots, x^{n}$ denoted by ${ }^{*} ; a, b, c, \ldots$ run from 1 to $p$ and $\alpha, \beta, \gamma, \ldots$ run from $p+1$ to $n$. The two parts of (21) are the metrics of $M_{1}^{p}(p \geq 2)$ and $M_{2}^{n-p}(n-p \geq 2)$ which are called the decompositions of the decomposable manifold

Let $\left(M^{n}, g\right)$ be a decomposable Riemannian manifold such that for $2 \leq p \leq n-2$. Here throughout this section each object denoted by a 'bar' is assumed to be from $M_{l}$ and each object denoted by a 'star' is assumed to be from $M_{2}$.

Let $\bar{X}, \bar{Y}, \bar{Z}, \bar{U}, \bar{V} \in \chi\left(M_{1}\right)$ and $\stackrel{*}{X}, \stackrel{*}{Y}, \stackrel{*}{Z}, \stackrel{*}{U}, V^{*} \in \chi\left(M_{2}\right), \chi\left(M_{i}\right)$ being the Lie algebra of smooth vector fields on $M_{i}, i=1,2$. Then we have the following relations [13]:

$$
\begin{aligned}
& R(\stackrel{*}{X}, \bar{Y}, \bar{Z}, \bar{U})=R(\bar{X}, \stackrel{*}{Y}, \bar{Z}, \stackrel{*}{U})=R(\bar{X}, \stackrel{*}{Y}, \stackrel{*}{Z}, \stackrel{*}{U})=0, \\
& \left(\nabla_{\dot{X}} R\right)(\bar{Y}, \bar{Z}, \bar{U}, \bar{V})=\left(\nabla_{\bar{X}} R\right)\left(\bar{Y},{ }_{Z}^{*}, \bar{U}, \stackrel{*}{V}\right)=\left(\nabla_{\dot{X}} R\right)(\bar{Y}, \stackrel{*}{Z}, \bar{U}, \stackrel{*}{V})=0 \text {, } \\
& R(\bar{X}, \bar{Y}, \bar{Z}, \bar{U})=\bar{R}(\bar{X}, \bar{Y}, \bar{Z}, \bar{U}), \\
& R(\stackrel{*}{X}, \stackrel{*}{Y}, \stackrel{*}{Z}, \stackrel{*}{U})=\stackrel{*}{R}(\stackrel{*}{X}, \stackrel{*}{Y}, \stackrel{*}{Z}, \stackrel{*}{U}), \\
& S(\bar{X}, \bar{Y})=\bar{S}(\bar{X}, \bar{Y}), \\
& S(\stackrel{*}{X}, \stackrel{*}{Y})=\stackrel{*}{S}(\stackrel{*}{X}, \stackrel{*}{Y}), \\
& \left(\nabla_{\bar{X}} S\right)(\bar{Y}, \bar{Z})=\left(\bar{\nabla}_{\bar{X}} S\right)(\bar{Y}, \bar{Z}), \\
& \left(\nabla_{\dot{X}} S\right)(\stackrel{*}{Y}, \stackrel{*}{Z})=\left(\stackrel{\nabla}{\nabla}_{\dot{X}}^{*} S\right)(\stackrel{*}{Y}, \stackrel{*}{Z}), \\
& r=\bar{r}+\stackrel{*}{r},
\end{aligned}
$$

where $r, \bar{r}$ and $\dot{r}$ are the scalar curvature of $M, M_{1}, M_{2}$ respectively.

Let us consider a Riemannian manifold $\left(M^{n}, g\right)$ which is decomposable $(P C R S)_{n}$. Then $M^{n}=M_{1}^{p} \times M_{2}^{n-p},(2 \leq p \leq n-2)$.

Now from (2), we have

$$
\begin{aligned}
& \left(\nabla_{\bar{X}} S\right)(\bar{Y}, \bar{Z})+\left(\nabla_{\bar{Y}} S\right)(\bar{Z}, \bar{X})+\left(\nabla_{\bar{Z}} S\right)(\bar{X}, \bar{Y}) \\
& =2 A(\bar{X}) S(\bar{Y}, \bar{Z})+A(\bar{Y}) S(\bar{X}, \bar{Z})+A(\bar{Z}) S(\bar{Y}, \bar{X}) \\
& \text { or, } \\
& \left(\nabla_{\bar{X}} S\right)(\bar{Y}, \bar{Z})+\left(\nabla_{\bar{Y}} S\right)(\bar{Z}, \bar{X})+\left(\nabla_{\bar{Z}} S\right)(\bar{X}, \bar{Y}) \\
& =2 A(\bar{Y}) S(\bar{Z}, \bar{X})+A(\bar{Z}) S(\bar{X}, \bar{Y})+A(\bar{X}) S(\bar{Y}, \bar{Z}) \\
& \text { or, } \\
& \left(\nabla_{\bar{X}} S\right)(\bar{Y}, \bar{Z})+\left(\nabla_{\bar{Y}} S\right)(\bar{Z}, \bar{X})+\left(\nabla_{\bar{Z}} S\right)(\bar{X}, \bar{Y}) \\
& =2 A(\bar{Z}) S(\bar{X}, \bar{Y})+A(\bar{X}) S(\bar{Y}, \bar{Z})+A(\bar{Y}) S(\bar{Z}, \bar{X}) \text {, }
\end{aligned}
$$


and

$$
\begin{aligned}
& \left(\nabla_{\dot{*}} S\right)(\stackrel{*}{Y}, \stackrel{*}{Z})+\left(\nabla_{\dot{*}} S\right)(\stackrel{*}{Z}, \stackrel{*}{X})+\left(\nabla_{Z} S\right)(\stackrel{*}{X}, \stackrel{*}{Y}) \\
& =2 A(\stackrel{*}{X}) S(\stackrel{*}{Y}, \stackrel{*}{Z})+A(\stackrel{*}{Y}) S(\stackrel{*}{X}, \stackrel{*}{Z})+A(\stackrel{*}{Z}) S(\stackrel{*}{Y}, \stackrel{*}{X}) \\
& \text { or, } \\
& \left(\nabla_{X} S\right)(\stackrel{*}{Y}, \stackrel{*}{Z})+\left(\nabla_{\dot{Y}} S\right)(\stackrel{*}{Z}, \stackrel{*}{X})+\left(\nabla_{\dot{Z}} S\right)(\stackrel{*}{X}, \stackrel{*}{Y}) \\
& =2 A(\stackrel{*}{Y}) S(\stackrel{*}{Z}, \stackrel{*}{X})+A(\stackrel{*}{Z}) S(\stackrel{*}{X}, \stackrel{*}{Y})+A(\stackrel{*}{X}) S\left(\stackrel{*}{Y}, \stackrel{*}{Z}^{\prime}\right) \\
& \text { or, } \\
& \left(\nabla_{\dot{X}} S\right)\left(\stackrel{*}{Y}, \stackrel{*}{Z}^{\prime}\right)+\left(\nabla_{\dot{Y}} S\right)(\stackrel{*}{Z}, \stackrel{*}{X})+\left(\nabla_{\dot{Z}} S\right)(\stackrel{*}{X}, \stackrel{*}{Y}) \\
& =2 A(\stackrel{*}{Z}) S(\stackrel{*}{X}, \stackrel{*}{Y})+A(\stackrel{*}{X}) S\left(\stackrel{*}{Y}, \stackrel{*}{Z}^{\prime}\right)+A(\stackrel{*}{Y}) S(\stackrel{*}{Z}, \stackrel{*}{X}) \text {. }
\end{aligned}
$$

From (22), we find

$$
\begin{gathered}
A(\stackrel{*}{X}) S(\bar{Y}, \bar{Z})=0, \\
A(\bar{X}) S(\stackrel{*}{Y}, \stackrel{*}{Z})=0 .
\end{gathered}
$$

Now from (24) it follows that either $A(\stackrel{*}{X})=0$ for any vector field $\stackrel{*}{X} \in \chi\left(M_{2}\right)$ or $S(\bar{Y}, \bar{Z})=0$ for all vector fields $\bar{Y}, \bar{Z} \in \chi\left(M_{1}\right)$, i.e., the decomposition $M_{l}$ is Ricci flat.

Again if $A(\stackrel{*}{X})=0$ then from $(23)$, we get

$$
\left(\nabla_{\dot{*}} S\right)(\stackrel{*}{Y}, \stackrel{*}{Z})+\left(\nabla_{\dot{*}} S\right)(\stackrel{*}{Z}, \stackrel{*}{X})+\left(\nabla_{Z} S\right)(\stackrel{*}{X}, \stackrel{*}{Y})=0,
$$

that is, the Ricci tensor of the decomposition $M_{2}$ is cyclic parallel.

Similarly from (25), we obtain either the Ricci tensor of the decomposition $M_{l}$ is cyclic parallel or the decomposition $M_{2}$ is Ricci flat. Thus, we can state the following:

Theorem 4.1. In a decomposable $(P C R S)_{n}$, one of the decompositions is Ricci flat and the Ricci tensor of the other decomposition is cyclic parallel.

\section{Totally Umbilical Hypersurfaces of $(P C R S)_{n}$}

Let $(\bar{V}, \bar{g})$ be an $(n+1)$-dimensional Riemannian manifold covered by a system of coordinate neighbourhoods $\left\{U, y^{\alpha}\right\}$. Let $(V, g)$ be a hypersurface of $(\bar{V}, \bar{g})$ defined in a locally coordinate system by means of a system of parametric equation $y^{\alpha}=y^{\alpha}\left(x^{i}\right)$, where Greek indices take values $1,2, \ldots, n$ and Latin indices take values $1,2, \ldots,(n+1)$. Let $N^{\alpha}$ be the components of a local unit normal to $(V, g)$. Then we have 


$$
\begin{aligned}
g_{i j} & =\bar{g}_{\alpha \beta} y_{i}^{\alpha} y_{j}^{\beta}, \\
\bar{g}_{\alpha \beta} N^{\alpha} y_{j}^{\beta} & =0, \bar{g}_{\alpha \beta} N^{\alpha} N^{\beta}=e=1, \\
y_{i}^{\alpha} y_{j}^{\beta} g^{i j} & =\bar{g}^{\alpha \beta}-N^{\alpha} N^{\beta}, y_{i}^{\alpha}=\frac{\partial y^{\alpha}}{\partial x^{i}} .
\end{aligned}
$$

The hypersurface $(V, g)$ is called a totally umbilical hypersurface $([14,15])$ of $(\bar{V}, \bar{g})$ if its second fundamental form $\Omega_{i j}$ satisfies

$$
\Omega_{i j}=H g_{i j}, y_{i, j}^{\alpha}=g_{i j} H N^{\alpha},
$$

where the scalar function $H$ is called the mean curvature of $(V, g)$ given by $H=\frac{1}{n} \Sigma g^{i j} \Omega_{i j}$. If, in particular, $H=0$, i.e.,

$$
\Omega_{i j}=0,
$$

then the totally umbilical hypersurface is called a totally geodesic hypersurface of $(\bar{V}, \bar{g})$.

The equation of Weingarten for $(V, g)$ can be written as $N_{, j}^{\alpha}=-\frac{H}{n} y_{j}^{\alpha}$. The structure equations of Gauss and Codazzi $([14,15])$ for $(V, g)$ and $(\bar{V}, \bar{g})$ are respectively given by

$$
\begin{aligned}
& R_{i j k l}=\bar{R}_{\alpha \beta \gamma \delta} B_{i j k l}^{\alpha \beta \gamma \delta}+H^{2} G_{i j k l}, \\
& \bar{R}_{\alpha \beta \gamma \delta} B_{i j k}^{\alpha \beta \gamma} N^{\delta}=H_{, i} g_{j k}-H_{, j} g_{i k},
\end{aligned}
$$

where $R_{i j k l}$ and $\bar{R}_{\alpha \beta \gamma \delta}$ are curvature tensors of $(V, g)$ and $(\bar{V}, \bar{g})$ respectively, and

$$
B_{i j k l}^{\alpha \beta \gamma}=B_{i}^{\alpha} B_{j}^{\beta} B_{k}^{\gamma} B_{l}^{\delta}, B_{i}^{\alpha}=y_{i}^{\alpha}, G_{i j k l}=g_{i l} g_{j k}-g_{i k} g_{j l} .
$$

Also we have $([14,15])$

$$
\begin{gathered}
\bar{S}_{\alpha \delta} B_{i}^{\alpha} B_{j}^{\delta}=S_{i j}-(n-1) H^{2} g_{i j}, \\
\bar{S}_{\alpha \delta} N^{\alpha} B_{i}^{\delta}=(n-1) H_{, i}, \\
\bar{r}=r-n(n-1) H^{2},
\end{gathered}
$$

where $S_{i j}$ and $\bar{S}_{\alpha \delta}$ are the Ricci tensors of $(V, g)$ and $(\bar{V}, \bar{g})$ respectively and $r$ and $\bar{r}$ are the scalar curvatures of $(V, g)$ and $(\bar{V}, \bar{g})$ respectively.

In terms of local coordinates the relation (2) can be written as

$$
\begin{aligned}
& S_{i j, k}+S_{j k, i}+S_{k i, j}=2 A_{k} S_{i j}+A_{i} S_{j k}+A_{j} S_{k i} \\
& \text { or, } \\
& S_{i j, k}+S_{j k, i}+S_{k i, j}=2 A_{i} S_{j k}+A_{j} S_{k i}+A_{k} S_{i j} \\
& \text { or, } \\
& S_{i j, k}+S_{j k, i}+S_{k i, j}=2 A_{j} S_{k i}+A_{k} S_{i j}+A_{i} S_{j k} .
\end{aligned}
$$




\section{S.K. Hui}

Let $(\bar{V}, \bar{g})$ be a $(P C R S)_{n}$. Then we get

$$
\begin{aligned}
& \bar{S}_{\alpha \beta, \gamma}+\bar{S}_{\beta \gamma, \alpha}+\bar{S}_{\gamma \alpha, \beta}=2 A_{\gamma} \bar{S}_{\alpha \beta}+A_{\alpha} \bar{S}_{\beta \gamma}+A_{\beta} \bar{S}_{\gamma \alpha} \\
& \text { or, } \\
& \bar{S}_{\alpha \beta, \gamma}+\bar{S}_{\beta \gamma, \alpha}+\bar{S}_{\gamma \alpha, \beta}=2 A_{\alpha} \bar{S}_{\beta \gamma}+A_{\beta} \bar{S}_{\gamma \alpha}+A_{\gamma} \bar{S}_{\alpha \beta} \\
& \text { or, } \\
& \bar{S}_{\alpha \beta, \gamma}+\bar{S}_{\beta \gamma, \alpha}+\bar{S}_{\gamma \alpha, \beta}=2 A_{\beta} \bar{S}_{\gamma \alpha}+A_{\gamma} \bar{S}_{\alpha \beta}+A_{\alpha} \bar{S}_{\beta \gamma} .
\end{aligned}
$$

where $A, B$ are nowhere vanishing 1-forms.

Multiplying both sides of (37) by $B_{i j k}^{\alpha \beta \gamma}$ and then using (33) and (36), we obtain either

$$
H=0
$$

or

$$
2\left[H_{, k} g_{i j}+H_{, i} g_{k j}+H_{, j} g_{i k}\right]=H\left[2 A_{k} g_{i j}+A_{i} g_{k j}+A_{j} g_{i k}\right]
$$

Transvecting (38) by $g^{i j}$, we obtain

$$
H_{, k}=\frac{n+1}{n+2} A_{k}
$$

for all $k$. This leads to the following:

Theorem 5.1. If the totally umbilical hypersurface of a $(P C R S)_{n}$ is a $(P C R S)_{n}$ then either the manifold is a totally geodesic hypersurface or the associated 1-form $A$ satisfies the relation (39).

We now consider that the space $(V, g)$ is totally geodesic hypersurface, i.e.,

$$
H=0 \text {. }
$$

In view of (40), (33) yields

$$
\bar{S}_{\alpha \delta} B_{i}^{\alpha} B_{j}^{\delta}=S_{i j}
$$

Using (41) in (37), we have the relation (36). Thus we can state the following:

Theorem 5.2. The totally geodesic hypersurface of a $(P C R S)_{n}$ is also $(P C R S)_{n}$.

\section{References}

[1] Patterson E.M., 1952. Some theorems on Ricci-recurrent spaces, Journal of London Mathematical Society, 27, 287-295.

[2] Szabo Z.I., 1982. Structure theorems on Riemannian spaces satisfying $R(X, Y) . R=0$, The local version, Journal of Differential Geometry, 17, 531-582.

[3] Chaki M.C., 1988. On pseudo Ricci symmetric manifolds, Bulgarian Journal of Physics, 15, 526-531.

[4] Shaikh A.A., Hui S.K., 2009. On pseudo cyclic Ricci symmetric manifolds, Asian-European Journal of Mathematics, 2 (2): 227-237. 
[5] Shaikh A.A., Hui S.K., 2010. On pseudo cyclic Ricci symmetric manifolds admitting semi-symmetric connection, Scientia, Series A: Mathematical Sciences, 20, 73-80.

[6] Shaikh A.A., Hui S.K., 2010. On pseudo cyclic Ricci symmetric spacetimes, Advanced Studies in Contemporary Mathematics, 20 (3): 425-432.

[7] Shaikh A.A., Hui S.K., 2011. Some global properties of pseudo cyclic Ricci symmetric manifolds, Applied Sciences, 13, 97-101.

[8] Özen F., Altay S., 2001. On weakly and pseudo symmetric Riemannian spaces, Indian Journal of Pure and Applied Mathematics, 33 (10): 1477-1488.

[9] Özen F., Altay S., 2008. On weakly and pseudo concircular symmetric structures on a Riemannian manifold, Acta Universitatis Palackianae Olomucensis Facultas Rerum Naturalium, Mathematica, 47, 129-138.

[10] Shaikh A.A., Roy I., Hui S. K., 2010. On totally umbilical hypersurfaces of weakly conharmonically symmetric spaces, Global Journal Science Frontier Research, 10 (4): 28-30.

[11] Khan Q., 2004. On recurrent Riemannian manifolds, Kyungpook Mathematical Journal, 44, 269-276.

[12] Ruse H.S., 1947. Three dimensional spaces of recurrent curvature, Proceedings of London Mathematical Society, 50 (2): 438-446.

[13] Yano K., Kon M., 1986. Structure on manifolds, Series in Pure Mathematics, Vol. 3, World Scientific Publishing, Singapore.

[14] Chen B.Y., 1973. Geometry of submanifolds, Marcel-Dekker, New York, p. 298.

[15] Eisenhart L.P., 1949. Riemannian Geometry, Princeton University Press, Princeton, New Jersey, p. 306. 